\begin{tabular}{|c|c|}
\hline Title & Optical nonlinearities of Se loaded zeolite (ZSM-5): A molded nanowire system \\
\hline Author(s) & Tanaka, Keiji; Saitoh, A kira \\
\hline Citation & $\begin{array}{l}\text { A pplied Physics Letters, 94(24), } 241905 \\
\text { https://doi.org/10.1063/1.3155452 }\end{array}$ \\
\hline Issue Date & 2009-06-15 \\
\hline Doc URL & http:/hdl.handle.net/2115/38749 \\
\hline Rights & $\begin{array}{l}\text { Copyright } 2009 \text { A merican Institute of Physics. This article may be downloaded for personal use only. A ny other use } \\
\text { requires prior permission of the author and the A merican Institute of Physics. }\end{array}$ \\
\hline Type & article \\
\hline File Information & 94_24_241905.pdf \\
\hline
\end{tabular}

Instructions for use 


\title{
Optical nonlinearities of Se-loaded zeolite (ZSM-5): A molded nanowire system
}

\author{
Keiji Tanaka ${ }^{\text {a) }}$ and Akira Saitoh \\ Department of Applied Physics, Graduate School of Engineering, Hokkaido University, \\ Sapporo 060-8628, Japan
}

(Received 14 April 2009; accepted 28 May 2009; published online 16 June 2009)

\begin{abstract}
Nonlinear optical properties of Se-loaded ZSM-5 single crystals have been studied comparatively with those of glassy Se. Two-photon absorption coefficients and intensity-dependent refractive-index of the Se-zeolite are higher by three orders of magnitude than those of glassy Se. The enhancements can be ascribed to electron confinement in single Se chains. (C) 2009 American Institute of Physics. [DOI: 10.1063/1.3155452]
\end{abstract}

Sufficiently high third-order optical nonlinear materials are being sought for fabrication of compact all-optical switches and so forth operating at the $1.5 \mu \mathrm{m}$ communication band. For instance, a waveguide switch with a length of $1 \mathrm{~cm}$ and a cross section of $10 \mu \mathrm{m}^{2}$ operating under $1 \mathrm{~W}$ light needs an intensity-dependent refractive index $n_{2}$ of $10^{-2} \mathrm{~cm}^{2} / \mathrm{GW}$. However, it becomes to be known that such a high nonlinearity is difficult to attain using homogeneous materials that are transparent at the wavelength of $1.5 \mu \mathrm{m} .{ }^{1-4}$ Then, our target is directed to heterogeneous media such as dye-doped polymers, ${ }^{5}$ particle-doped inorganic media, ${ }^{6,7}$ and photonic crystals. ${ }^{8}$

We here explore nonlinear properties of a chalcogenloaded zeolite, a kind of inorganic-dye-doped systems. This guest-host composite appears to be promising as nonlinear materials with the following reasons. It has been demonstrated that the chalcogenide glass, due to its smaller energy gap than that of the oxide, ${ }^{6}$ possesses higher nonlinearity of $n_{2} \leq 10^{-3} \mathrm{~cm}^{2} / \mathrm{GW} .^{1,2,9}$ However, the nonlinearity is still insufficient for practical uses. In addition, the glass is more damageable ${ }^{10}$ than the oxide due to its lower glass-transition temperature. Hence, it is intriguing to employ the zeolite, a porous silica-based crystal, ${ }^{11}$ as a host for incorporating the chalcogen. In addition, recent studies have produced submillimeter size zeolite single crystals, ${ }^{12}$ suitable to nonlinear optical measurements. This letter reports optical nonlinearity of Se-loaded ZSM-5 ( $z$-Se) in comparison with that of glassy $\mathrm{Se}(\mathrm{g}$-Se). Fundamental properties of Se-loaded ZSM-5 have been reported elsewhere. ${ }^{13}$

Two kinds of samples were investigated. One was $z$-Se, the preparation detail being reported in Ref. 13. In short, ZSM-5 crystals with a typical dimension of $40 \times 40$ $\times 200 \mu \mathrm{m}^{3}$ for the $a, b$, and $c$ axes (see Fig. 1) were employed as matrices and $6 \mathrm{~N}$ purity Se was thermally incorporated from gas phases in vacuum. Varied preparation conditions produced samples with colors of yellow, orange, and brown. And, the present work mostly dealt with the orange sample, for which thermal desorption measurements suggested that Se concentration in the sample was $\sim 10^{14}$ atoms or $\sim 10^{20} / \mathrm{cm}^{3}$. The $z$-Se sample was laid on an aperture with a diameter of $25 \mu \mathrm{m}$, and probe light was propagated through the sample along the zeolite $b$-axis. Light polarization was not resolved. The other sample was $g$-Se, the bulk

${ }^{a)}$ Electronic mail: keiji@eng.hokudai.ac.jp. and films being prepared through the conventional meltquenching method and vacuum evaporation. ${ }^{14}$

Optical transmission spectra of these samples were evaluated using a laser-detector system. ${ }^{13}$ Two-photon absorption coefficient $\beta$ and intensity-dependent refractive index $n_{2}$ were measured using three pulsed sources: a 5 ns yttrium aluminum garnet (YAG) laser, an optical parametric oscillator (OPO), and a 0.3 ns YAG laser. Optical setups employed were two kinds. $\beta$ spectra were evaluated through intensity-dependent transmittance measurements. ${ }^{1,15}$ For measuring $\beta$ and $n_{2}$ at $\lambda \simeq 1064 \mathrm{~nm}$, we employed a $z$-scan technique ${ }^{1,16}$ in combination with a profile imager, ${ }^{17}$ which monitored intensity patterns of transmitting light. Through fitting Gaussian curves to the patterns, which were averaged over repeated light pulses, we could evaluate the total light intensity and central peak intensity. These values correspond to the so-called open- and closed-aperture signals. ${ }^{1,16}$ This profile-imaging $z$-scan method can greatly reduce noises, which is important for somewhat irregular samples as the zeolite. $^{17}$

Figure 1 shows transmission spectra of pure ZSM-5 and $z$-Se with photographs of typical samples. ZSM-5 is transparent over wavelengths of $\lambda \simeq 350-1064 \mathrm{~nm}$ with a slight

\section{Photon energy (eV)}

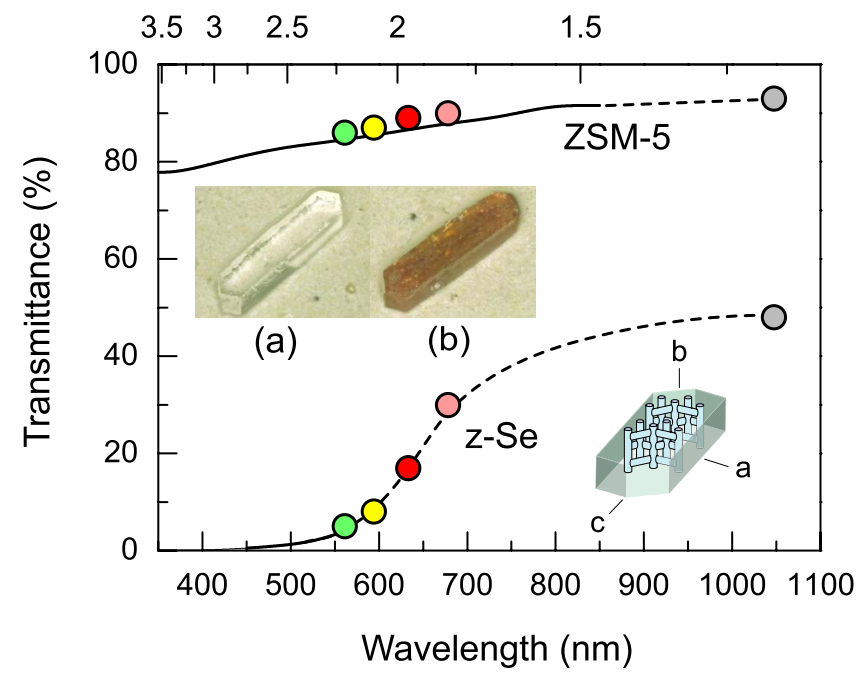

FIG. 1. (Color online) Transmission spectra (lines with circles) of typical ZSM-5 and $z$-Se. Insets give photographs of (a) ZSM-5 and (b) $z$-Se and an illustration of ZSM-5, with crystal axes and magnified channels. 


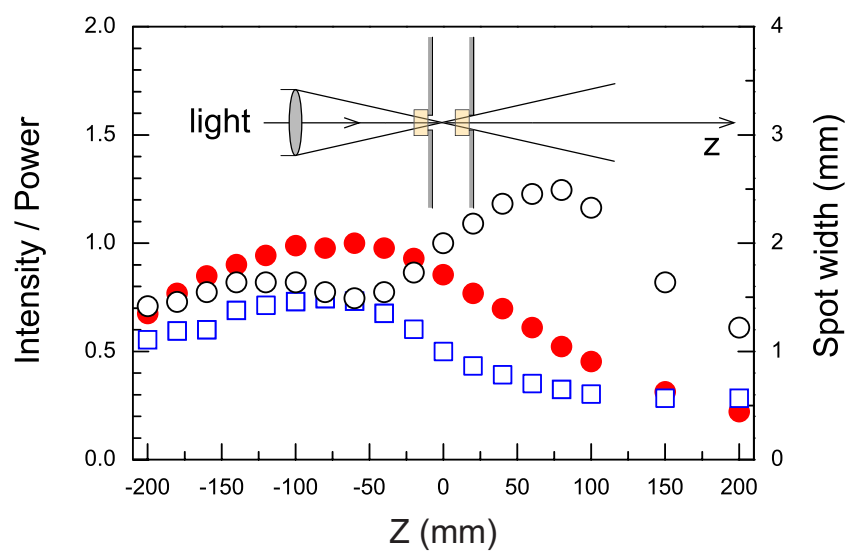

FIG. 2. (Color online) A result of the imaging $z$-scan measurement for $z$-Se, with an inset showing light-focusing geometry for the two positions of a sample (brown $z$-Se on an aperture) at $z<0$ and $z>0$. Open (black) and solid (red) circles show the central and the total intensity, being normalized on the left-hand side axis, which correspond to the closed- and openaperture signals, and open (blue) squares show the spot width with the right-hand side axis.

transmission decrease at shorter wavelengths, which may reflect light scattering due to minute cracks. On the other hand, $z-$ Se is opaque at $\lambda \lesssim 600 \mathrm{~nm}^{13}$

Figure 2 shows a result of the imaging $z$-scan measurement for a $z$-Se sample at $\lambda \simeq 1064 \mathrm{~nm}$, pulse width of $5 \mathrm{ns,}$ and irradiance of $\sim 0.5 \mathrm{GW} / \mathrm{cm}^{2}$. We see for the central intensity (open circles), which corresponds to the closedaperture signal, a valley-peak distance of $\sim 140 \mu \mathrm{m}$, and a normalized valley-peak transmittance of $\Delta T_{\mathrm{p}-\mathrm{v}} \simeq 0.3$, which gives $n_{2} \simeq 10^{-3} \mathrm{~cm}^{2} / \mathrm{GW}$ with sample-to-sample variations of a half order. Dependence upon the pulse widths $(0.3$ and $5 \mathrm{~ns}$ ) has been smaller than this variation. The profile asymmetry and the intensity reductions at $z \simeq \pm 200 \mu \mathrm{m}$ reflect the eclipse due to the small aperture (see, the inset in Fig. 2). On the other hand, the total power, which corresponds to the open-aperture signal, shows a peak at $z \simeq-60 \mu \mathrm{m}$, not at $z \simeq 0 \mu \mathrm{m}$, possibly resulting from the eclipse. The twophoton absorption coefficient $\beta$ at this wavelength is smaller than a detection limit. We also see that the Gaussian diameter of the light spot shows a peak at $z \simeq-70 \mu \mathrm{m}$, which probably reflects a beam-defocusing effect arising from the positive $n_{2}$.

Figure 3(a) compares linear absorption spectra of $g$-Se and $z$-Se. The linear absorption spectrum of $g$-Se is mostly consistent with previous results, ${ }^{14}$ giving an optical gap $E_{g}$ of $\sim 2.0 \mathrm{eV}$. On the other hand, $z$-Se shows a less-steep absorption edge. It is plausible that this gradual slope arises from structural inhomogeneity, i.e., Se is impregnated as a form of single chains with an average chain length of $10^{2}$ atoms, which possibly varies from site to site. ${ }^{13}$ Then, defining the optical gap as a photon energy at the absorption coefficient $\alpha$ of $10^{3} \mathrm{~cm}^{-1}$, we have $E_{g} \simeq 2.2 \mathrm{eV}$, which blueshifts by $\sim 0.2 \mathrm{eV}$ from the bulk gap.

Figure 3(a) also compares $\beta$ spectra of $g$-Se and $z$-Se, which have been evaluated from intensity-dependent transmittances of light pulses emitted from the OPO. ${ }^{1,15}$ Different from the linear spectra, the $\beta$ spectra seem to have peaks at $\sim 1.5$ and $\sim 2.2 \mathrm{eV}$ in $g$-Se and $z$-Se, the positions being located between $E_{g} / 2$ and $E_{g}$ in agreement with theoretical predictions. ${ }^{3,4}$ We here underline that $z$-Se shows higher $\beta$ than that of $g$-Se by approximately three orders. It should also be mentioned that a brown-color $z$-Se has exhibited a

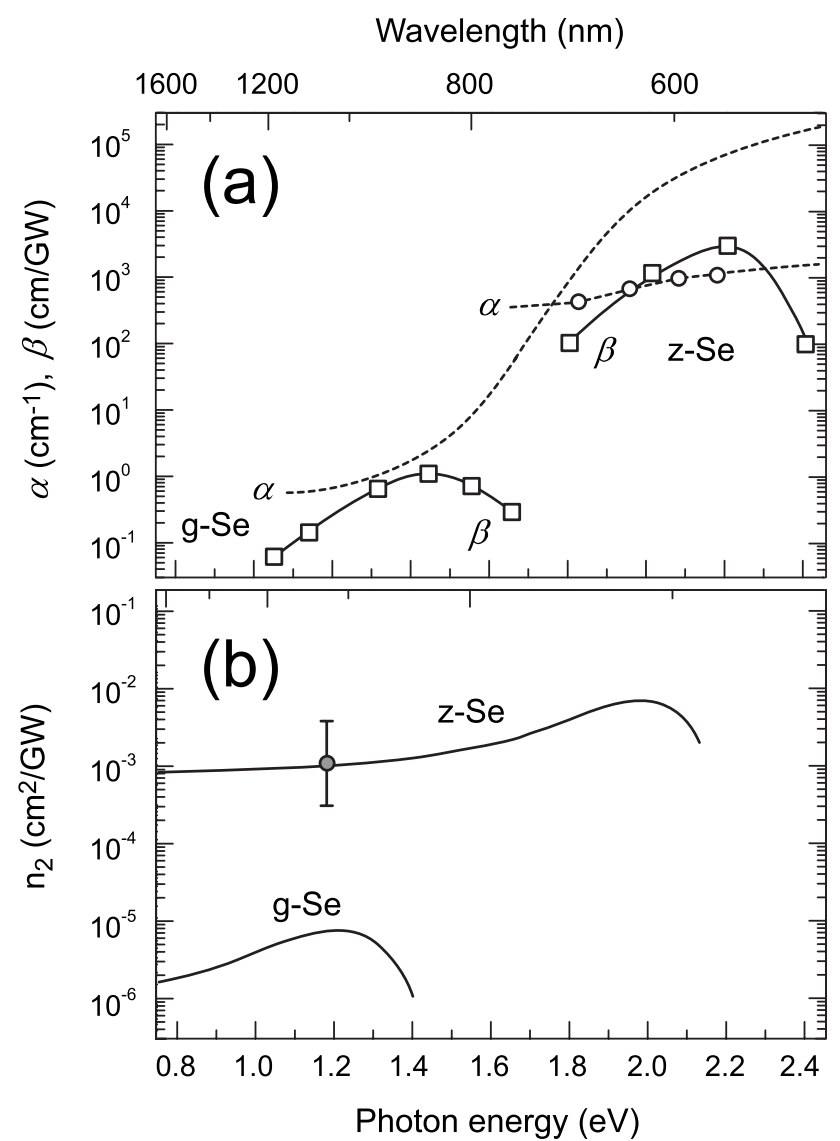

FIG. 3. (a) $\alpha$ (dashed lines) and $\beta$ (squares) spectra for $g$-Se and $z$-Se, and approximated Gaussian curves (solid lines) and (b) calculated $n_{2}$ curves and $n_{2}$ at $1.17 \mathrm{eV}(\lambda=1064 \mathrm{~nm})$ for $z-S e$.

still greater $\beta$ value $\left(\lesssim 10^{5} \mathrm{~cm} / \mathrm{GW}\right){ }^{18}$ A damage threshold of $z$-Se has appeared to be higher by an order than that $\left(\sim 0.5 \mathrm{GW} / \mathrm{cm}^{2}\right)$ of $g$-Se.

Using these $\beta$ spectra, we can estimate $n_{2}$ spectra, as shown by solid curves in Fig. 3(b). In this calculation, the $\beta$ spectra have been approximated by the Gaussian curves, as shown in Fig. 3(a), which are transformed using a nonlinear Kramers-Krönig relationship. ${ }^{19}$ On the other hand, as plotted in the figure, the $z$-scan measurement for $z$-Se gives $n_{2}$ of $10^{-3} \mathrm{~cm}^{2} / \mathrm{GW}$ at $1064 \mathrm{~nm}$, which is consistent with the calculated result. $n_{2}$ in $g$-Se at the wavelength could not be inspected, while it is predicted to be substantially smaller, as shown in Fig. 3(b).

Why does $z$-Se give such a greater nonlinearity than that of $g$-Se? We consider four possibilities. First, it is theoretically predicted that a material having smaller $E_{g}$ possesses a higher $n_{2}, 3,4$ but $z$-Se has a wider $E_{g}(\sim 2.2 \mathrm{eV})$ than that in $g$-Se $(\sim 2.0 \mathrm{eV})$. Second, we may envisage some effects arising from modifications of chemical bonds. Specifically, the electron wave function of the valence band, consisting of lone-pair electron states, is likely to more localize in $z$-Se. ${ }^{20}$ However, if such a modification can explain the enhancement of the three orders is questionable. Third, it is tempting to envisage exciton-confinement effects predicted for semiconductor-doped glasses. ${ }^{2}$ Nevertheless, if the theory, which presumes spherical semiconductor particles, can be applied to the present case is not known. Actually, the exciton Bohr radius $r_{B}$ in hexagonal $\mathrm{Se}$ is reported to be $\sim 1 \mathrm{~nm},{ }^{21}$ the Se chain radius $R$ is $\sim 0.3 \mathrm{~nm},{ }^{14}$ and the chain length $L$ in ZSM-5 is estimated at $\sim 10^{1} \mathrm{~nm}$ 
$\left(\sim 10^{2}\right.$ atoms $),{ }^{13}$ so that $r_{B} / R>1$ and $r_{B} / L<1$. Lastly, the most attractive interpretation is to follow a concept developed for one-dimensional quantum wells, in which the thirdorder polarizability increases in proportion to $L^{5}$, where $L$ is a well length. ${ }^{1,22}$ The single Se chain may be regarded as a one-dimensional quantum well and such a length enhancement is likely to exist. Actually, a preliminary experiment for $\mathrm{Se}_{2}$ anions in cancrinite ${ }^{23}$ could not detect $z$-scan signals, which is consistent with this model. We then expect that longer Se chains in zeolite will afford practical $n_{2}$ values for compact all-optical switches, keeping in mind that an implicit assumption in the quantum-well model is a longer mean-free path of electrons than $L$.

In conclusion, we have demonstrated that $\beta$ and $n_{2}$ and in $z$-Se reach, respectively, to $10^{3} \mathrm{~cm} / \mathrm{GW}$ at visible wavelengths and $10^{-3} \mathrm{~cm}^{2} / \mathrm{GW}$ at near infrared, which are much greater than those in glassy Se. Taking the wider optical gap in $z$-Se than those in glassy $\mathrm{Se}$, we suggest that the chalcogen-zeolite system is promising for fabricating compact optical devices, not only of the third-order but also of the second-order nonlinearity. ${ }^{23,24}$

The authors would like to thank Dr. Y. Kiyozumi for supplying ZSM-5 crystals, Dr. V. V. Poborchii for cancrinite-Se samples, Dr. A. V. Kolobov for private correspondences, and Mr. N. Minamikawa for experimental assistance. The authors also acknowledge financial support from Nippon Sheet Glass Foundation for Material Science and Engineering.

${ }^{1}$ R. W. Boyd, Nonlinear Optics, 2nd ed. (Academic, Amsterdam, 2003).

${ }^{2}$ M. Yamane and Y. Asahara, Glasses for Photonics (Cambridge University
Press, Cambridge, 2000), Chap. 4.

${ }^{3}$ M. Sheik-Bahae, D. J. Hagen, and E. W. Van Stryland, Phys. Rev. Lett. 65, 96 (1990)

${ }^{4}$ K. Tanaka, J. Phys. Chem. Solids 68, 896 (2007).

${ }^{5}$ H. Kishida, K. Hirota, H. Okamoto, H. Kokubo, and T. Yamamoto, Appl. Phys. Lett. 92, 033309 (2008).

${ }^{6}$ L. A. Gómez, F. E. P. dos Santos, A. S. L. Gomes, C. B. de Araŭjo, L. R. P. Kassab, and W. G. Hora, Appl. Phys. Lett. 92, 141916 (2008).

${ }^{7}$ L. Irimpan, V. P. N. Nampoori, P. Radhakrishnan, B. Krishnan, and A. Deepthy, J. Appl. Phys. 103, 033105 (2008).

${ }^{8}$ A. Ganany-Padowicz, I. Juwiler, O. Gayer, A. Bahabad, and A. Arie, Appl. Phys. Lett. 94, 091108 (2009).

${ }^{9}$ K. Ogusu, J. Yamasaki, S. Maeda, M. Kitao, and M. Minakata, Opt. Lett. 29, 265 (2004).

${ }^{10}$ K. Fedus, G. Boudebs, C. B. de Araújo, M. Cathelinaud, F. Charpentier, and V. Nazabal, Appl. Phys. Lett. 94, 061122 (2009).

${ }^{11}$ See http://www.iza-structure.org/databeses/pdf.htm for a comprehensive database of the zeolite.

${ }^{12}$ S. Shimizu and H. Hamada, Microporous Mesoporous Mater. 48, 39 (2001).

${ }^{13}$ A. Saitoh and K. Tanaka, J. Appl. Phys. 105, 113530 (2009).

${ }^{14}$ Selenium, edited by R. A. Zingaro and W. C. Cooper (Van Nostrand Reinhold, New York, 1974), Chaps. 3-5.

${ }^{15}$ K. Tanaka, Appl. Phys. Lett. 80, 177 (2002).

${ }^{16}$ M. Sheik-Bahae, A. A. Said, T. H. Wei, D. J. Hagen, and E. W. Van Stryland, IEEE J. Quantum Electron. 26, 760 (1990).

${ }^{17}$ N. Minamikawa and K. Tanaka, Jpn. J. Appl. Phys., Part 2 45, L960 (2006)

${ }^{18}$ However, $n_{2}$ of this sample could not be evaluated at $\lambda \simeq 1064 \mathrm{~nm}$, probably due to higher linear absorption and light scattering.

${ }^{19}$ K. Tanaka, J. Mater. Sci.: Mater. Electron. 16, 633 (2005).

${ }^{20}$ T. Yamaguchi and F. Yonezawa, J. Non-Cryst. Solids 156, 268 (1993).

${ }^{21}$ G. G. Roberts, S. Tutihasi, and R. C. Keezer, Phys. Rev. 166, 637 (1968).

${ }^{22}$ K. C. Rustagi and J. Ducuing, Opt. Commun. 10, 258 (1974).

${ }^{23}$ V. V. Poborchii, G.-G. Lindner, and M. Sato, J. Chem. Phys. 116, 2609 (2002).

${ }^{24}$ L. Werner, J. Caro, G. Finger, and J. Kornatowski, Zeolites 12, 658 (1992) 